\title{
The van der Waals Potential between Metastable Atoms and Solid Surfaces: Novel Diffraction Experiments versus Theory
}

\author{
Rüdiger Brühl, Peter Fouquet, Robert E. Grisenti, and J. Peter Toennies \\ Max-Planck-Institut für Strömungsforschung, Bunsenstr. 10, 37073 Göttingen, Germany
}

\author{
Gerhard C. Hegerfeldt, Thorsten Köhler, Martin Stoll, and Christian Walter \\ Institut für Theoretische Physik, Universität Göttingen, Bunsenstr. 9, 37073 Göttingen, Germany
}

\begin{abstract}
Highly polarizable metastable $\mathrm{He}^{*}\left(2^{3} \mathrm{~S}\right)$ and $\mathrm{Ne}^{*}\left(2^{3} \mathrm{P}\right)$ atoms have been diffracted from a $100 \mathrm{~nm}$ period silicon nitride transmission grating and the van der Waals coefficients $C_{3}$ for the interaction of the excited atoms with the silicon nitride surface have been determined from the diffraction intensities out to the 10th order. The results agree with calculations based on the non-retarded Lifshitz formula.
\end{abstract}

PACS numbers: 34.50.Dy, 03.75.Be

The van der Waals (vdW) force between atoms, molecules and solid surfaces is of far reaching importance in many branches of physics, chemistry, and biology [1]. For larger distances, retardation due to the exchange of virtual photons has to be included, while for distances much smaller than the smallest wavelength a non-retarded approach can be used. The theoretical foundations for atom-surface interactions were laid in the pioneering work of Lifshitz [2]. In this case the non-retarded $\mathrm{vdW}$ potential has the form $-C_{3} / l^{3}$ in leading order, where $l$ is the atom-surface separation and $C_{3}$ depends on the atom, its electronic state, and on the electronic states of the solid.

For groundstate rare gas atoms the $C_{3}$ coefficients have recently been measured with good accuracy [3]. Less is known about the van der Waals interactions of electronically excited metastable and Rydberg atoms, in particular the $C_{3}$ coefficient is not accurately known. Some time ago, transmission through narrow channels [4] and level shifts in closed or semi-infinite cavities [5,6] have been studied. Recently, inelastic electronic transitions on passage over a metal edge [7] and reflection from surfaces and (reflection) gratings [8] have been measured. Currently there is great interest in these potentials, in particular for metastable helium which is widely used in atom optics [9] as well as in surface physics [10] and for which Bose-Einstein condensation has recently been achieved [11]. The atom-surface van der Waals potentials could soon become relevant in guiding slow metastable rare gas atoms along microstructures [12] or in studying collective effects of Bose-Einstein condensed metastable He* atoms in contact with a surface.

From a theoretical point of view atoms in excited states are of particular interest. Their polarizability $\alpha$ is expected to increase as $n^{7}$, with correspondingly much stronger interactions [13]. Therefore it is not obvious whether approximate formulae for the groundstate atom-surface vdW potential are still applicable for excited atoms. Moreover, with the much stronger vdW interaction new effects such as higher multipole coefficients 14] can be expected.

In this article, an effective but simple experimental method is used to determine the atom-surface vdW coefficient $C_{3}$ for metastable rare gas atoms. It is based on diffraction of an atomic beam from a nanostructured transmission grating with a period of only $100 \mathrm{~nm}$. Modifications in the hierarchy of the intensities of the higher order maxima in the diffraction pattern have been shown to be directly related to the strength $C_{3}$ of the atom-surface vdW potential. In these experiments only the non-retarded regime is probed since the slit widths of about $50 \mathrm{~nm}$ are less than the distance at which retardation effects become significant.

The main difference to the case of rare gas groundstate atoms is the fact that for the theoretical evaluation of the experimental data the very convenient notion of an "effective" slit width can no longer be employed, due to the much stronger surface interaction. Therefore a new approach is presented which can also be applied to other atoms and molecules and materials 15 .

The diffraction apparatus has already been described in Ref. [16]. The metastable atoms are produced by a discharge in the free-jet expansion zone inside a sapphire nozzle (aperture diameter $160 \mu \mathrm{m}$ ) [17]. The He* in the beam is $98 \%$ in the ${ }^{3} \mathrm{~S}_{1}$ state and $2 \%$ in the ${ }^{1} \mathrm{~S}_{0}$ state [17] and the $\mathrm{Ne}^{*}$ is $85 \%$ in the ${ }^{3} \mathrm{P}_{2}$ state and $15 \%$ in the ${ }^{3} \mathrm{P}_{0}$ state [18]. The stable operation of the discharge limits the source pressure $P_{0}$ to the range $0.5-3 \mathrm{bar}$, with the consequence that the atomic beams have a rather broad velocity distribution with $\Delta v / u \simeq 13.3 \%$, where $\Delta v$ and $u$ denote the full half width and the mean velocity, respectively. The effective velocity spread could be reduced to about $\Delta v / u \simeq 3 \%$ by extracting the diffraction pattern for a given flight time "slice" through time-of-flight (TOF) spectra measured at closely spaced diffraction angles. After passing through the $0.72 \mathrm{~mm}$ diameter skimmer the beam is collimated by two $5 \mathrm{~mm}$ high, 20 micron (150 $\mathrm{mm}$ from the source) and 10 micron (1000 $\mathrm{mm}$ from the source) wide slits, before illuminating about $N=100$ slits of the silicon nitride transmission diffraction grating with a calibrated period of $100 \mathrm{~nm}$, placed $150 \mathrm{~mm}$ behind the second collimation slit. The depth $t=53 \mathrm{~nm}$ and wedge angle $\beta=11^{\circ}$ of the 
trapezoidally shaped grating bars were determined from transmission measurements [16] and the slit width at infinite velocity was measured to be $s_{0}=66.8 \mathrm{~nm}$ [3]. Although the relative population of metastable atoms is only about $10^{-5}$ for both $\mathrm{He}^{*}$ and $\mathrm{Ne}^{*}$ the groundstate atoms are entirely suppressed by the channel electron multiplier detector, mounted at a distance of $730 \mathrm{~mm}$ from the grating. The diffraction pattern is recorded by rotating the channeltron in angular steps of about $0.1 \mu \mathrm{rad}$ with respect to an axis passing through the grating bars. The $25 \mu \mathrm{m}$ wide detector slit, $r=430 \mathrm{~mm}$ downstream from the grating, provides an angular resolution of $100 \mu \mathrm{rad}$. TOF distributions were measured at each detector position for $5\left(\mathrm{He}^{*}\right)$ and 8 minutes $\left(\mathrm{Ne}^{*}\right)$. The peak shapes were fitted with a Gaussian to determine the peak areas $I_{n}$, which were normalized to the total over all peaks $I_{\text {tot }}$. Fig. 1 shows typical angular distributions for He* at a time slice corresponding to $2347 \mathrm{~m} / \mathrm{s}$ (de Broglie wavelength $\lambda=42.5 \mathrm{pm}$ ) and for $\mathrm{Ne}^{*}$ at $873 \mathrm{~m} / \mathrm{s}(\lambda=22.6 \mathrm{pm})$.

In the Fraunhofer limit $r \gg d$ the intensities of the $n$th order maxima in the diffraction pattern are given by

$$
I_{n} \propto\left|\frac{\sin \left(\frac{N k d}{2} \sin \vartheta_{n}\right)}{\sin \left(\frac{k d}{2} \sin \vartheta_{n}\right)} f_{\text {slit }}\left(\vartheta_{n}\right)\right|^{2},
$$

where the atom momentum is $m v=\hbar k=\frac{h}{\lambda}$ and the $n$th order diffraction angles are given by $\sin \vartheta_{n}=\frac{n \lambda}{d}$. At high velocities (small $\lambda$ ) small anomalies in the observed peak shapes at higher diffraction orders, which were attributed to Fresnel effects, were shown to have no significant influence on the interpolated Gaussian peak shapes. The slit function $f_{\text {slit }}(\vartheta)$ has the form [3]

$$
f_{\text {slit }}(\vartheta)=\frac{2 \cos \vartheta}{\sqrt{\lambda}} \int_{0}^{s_{0} / 2} \mathrm{~d} \zeta \cos \left[k \sin \vartheta\left(\frac{s_{0}}{2}-\zeta\right)\right] \tau(\zeta),
$$

where the integration is over half the slit opening from the edge $(\zeta=0)$ to the center $\left(\zeta=s_{0} / 2\right)$ with $\zeta$ as impact parameter with respect to the grating bar edge. In the usual Eikonal approximation [19] the amplitude $\tau(\zeta)$ at different positions in the slit becomes

$$
\tau(\zeta)=\exp \left(-\frac{\mathrm{i}}{\hbar v} \int_{-\infty}^{+\infty} \mathrm{d} z V_{\text {att }}(z, \zeta)\right),
$$

where the $z$-axis is in the beam direction. The attractive potential $V_{\text {att }}$ is $-C_{3} / l^{3}$ for a plane. For a grating bar, due to its finite extent, minor corrections occur which are taken into account here. Integration along a straight line trajectory for a given $\zeta$ yields, after some calculation,

$$
\tau(\zeta)=\exp \left(\frac{\mathrm{i} C_{3} t}{\hbar v \zeta^{3}} \frac{1+\frac{t}{2 \zeta} \tan \beta}{\left(1+\frac{t}{\zeta} \tan \beta\right)^{2}}\right) .
$$

Because of the much larger value of $C_{3}$ the cumulant expansion of Eq. (2) used in Ref. [3] was found not to converge for metastables so that the convenient notion of an effective slit width and the formula of Ref. [3] for $I_{n} / I_{\text {tot }}$ are no longer applicable. Thus it was necessary to calculate $I_{n} / I_{\text {tot }}$ from Eqs. (11)-(何) with $C_{3}$ as a parameter and use a least-square fit to the experimental values. This procedure is more complicated and more sensitive to experimental and numerical errors than that based on effective slit widths. Unlike Ref. [3] the best fit was obtained without a Debye-Waller damping factor to account for surface roughness [16]. The lack of sensitivity to these defects for He* and $\mathrm{Ne}^{*}$ is attributed to the greater range of the potential, so that their effect is smeared out.

The experimental results $C_{3}\left(\mathrm{He}^{*}\right)=(4.1 \pm 1.0) \mathrm{meV} \mathrm{nm}^{3}$ and $C_{3}\left(\mathrm{Ne}^{*}\right)=(2.8 \pm 1.0) \mathrm{meV} \mathrm{nm}^{3}$ are, as expected, more than an order of magnitude larger than the corresponding values of $C_{3}(\mathrm{He})=(0.10 \pm 0.02) \mathrm{meV} \mathrm{nm}^{3}$ and $C_{3}(\mathrm{Ne})=(0.21 \pm 0.04) \mathrm{meV} \mathrm{nm}^{3}$ for the groundstate atoms [3].

Present approximations of atom-surface vdW forces are based on the expression of Lifshitz [2],

$$
C_{3}=\frac{\hbar}{4 \pi} \int_{0}^{\infty} \mathrm{d} \omega \alpha(\mathrm{i} \omega) g(\mathrm{i} \omega),
$$

where $\alpha(\mathrm{i} \omega)$ is the dynamic polarizability of the atom and $g(\mathrm{i} \omega)$ is the corresponding response of the electrons of the solid which is related to the dielectric function $\epsilon$ by

$$
g(\mathrm{i} \omega)=\frac{\epsilon(\mathrm{i} \omega)-1}{\epsilon(\mathrm{i} \omega)+1} .
$$


Unfortunately, $\alpha$ and $g$ are not known in general. Vidali and Cole 21] have studied a model in which the partners are treated as single oscillators of frequencies $E_{\mathrm{a}} / \hbar$ for the atom and $E_{\mathrm{S}} / \hbar$ for the solid, i.e.

$$
\alpha(\mathrm{i} \omega) \approx \frac{\alpha(0)}{1+(\hbar \omega)^{2} / E_{\mathrm{a}}^{2}}
$$

and

$$
g(\mathrm{i} \omega) \approx \frac{g_{0}}{1+(\hbar \omega)^{2} / E_{\mathrm{S}}^{2}} .
$$

It has been shown earlier by Tang [22] that the one-oscillator approximation Eq. (7) is correct to within a few percent for the metastable atoms under consideration, and that

$$
E_{\mathrm{a}}=\frac{4 C_{6}}{3 \alpha^{2}(0)},
$$

where $C_{6}$ is the interatomic vdW coefficient which is well known for $\mathrm{He}^{*}$ and $\mathrm{Ne}^{*}$ from recent calculations 23 25] and gives $E_{a}=1.18 \mathrm{eV}$ for $\mathrm{He}^{*}$ and $E_{a}=2.04 \mathrm{eV}$ for $\mathrm{Ne}^{*}$. The static polarizability $\alpha(0)$ for $\mathrm{He}^{*}$ and $\mathrm{Ne}^{*}$ is given in the standard literature as $\alpha(0)=46.8 \AA^{3}$ and $\alpha(0)=27.6 \AA^{3}$, respectively. For the grating material, $g_{0}$ and $E_{\mathrm{S}}$ are not known, but $g(\mathrm{i} \omega)$ can be determined via Eq. (6) and Kramers-Kronig relations [26], once the imaginary part $\epsilon_{2}(\omega)$ of the dielectric function is given.

From optical measurements [27] on the low-pressure chemical vapor deposited (LPCVD) silicon nitride material of the grating in use, $\epsilon_{2}(\omega)$ has been determined from $1 \mathrm{eV}$ to about $6 \mathrm{eV}$. It has been shown recently that for LPCVD $\mathrm{SiN}_{x} \epsilon_{2}(\omega)$ over all frequencies is essentially given by the Tauc-Lorentz formula 28]

$$
\epsilon_{2}(\omega)=\Theta\left(\omega-\Omega_{\mathrm{T}}\right) \frac{A \Omega \Gamma\left(\omega-\Omega_{\mathrm{T}}\right)^{2}}{\left[\left(\omega^{2}-\Omega^{2}\right)^{2}+\Gamma^{2} \omega^{2}\right] \omega},
$$

where $\Theta$ is the step function, $\hbar \Omega_{\mathrm{T}}$ represents the optical band gap of the material, $A, \Omega, \Gamma$ are the strength, frequency, and spectral width, respectively, of the characteristic electronic transitions within the solid. The $\epsilon_{2}(\omega)$ that results from the optical measurements [27] is perfectly described by Eq. (10), with $\hbar \Omega_{\mathrm{T}}=2.29 \mathrm{eV}, \hbar A=74.5 \mathrm{eV}, \hbar \Omega=7.17 \mathrm{eV}$, and $\hbar \Gamma=7.62 \mathrm{eV}$.

With the response function $g(i \omega)$ of the solid determined from $\epsilon_{2}(\omega)$ as described above and using for $\alpha(i \omega)$ the approximate Eq. (7), the Lifshitz formula Eq. (5) yields $C_{3}\left(\mathrm{He}^{*}\right)=(3.9 \pm 0.1) \mathrm{meV} \mathrm{nm}{ }^{3}$ and $C_{3}\left(\mathrm{Ne}^{*}\right)=(3.6 \pm$ $0.1) \mathrm{meV} \mathrm{nm}^{3}$, in agreement within errors with the present experimental values $C_{3}\left(\mathrm{He}^{*}\right)=(4.1 \pm 1.0) \mathrm{meV} \mathrm{nm}^{3}$ and $C_{3}\left(\mathrm{Ne}^{*}\right)=(2.8 \pm 1.0) \mathrm{meV} \mathrm{nm}^{3}$.

Since for He* the dynamical polarizability at imaginary frequencies $\alpha(\mathrm{i} \omega)$ is known over nearly the entire frequency range from theoretical calculations [23,29], the single oscillator approximation Eq. (7) can be checked with the more exact $\alpha$ in Eq. (5) which leads to $C_{3}\left(\mathrm{He}^{*}\right)=(4.0 \pm 0.1) \mathrm{meV} \mathrm{nm}^{3}$ - only a $3 \%$ correction, as expected from Ref. 22].

Identifying in Eq. (8) the oscillator strength $g_{0}$ with the static limit $g(\mathrm{i} \omega \rightarrow 0)=0.588$ of Eq. (8) that is extracted from the optical data, and the theoretical values of $C_{3}$ are reproduced to within less than $10 \%$ by the formula [21].

$$
C_{3}^{1 \text { osc }}=\alpha(0) g_{0} \frac{E_{\mathrm{a}} E_{\mathrm{S}}}{8\left(E_{\mathrm{a}}+E_{\mathrm{S}}\right)},
$$

which corresponds to Eq. (5) with the one-oscillator approximations, Eqs. (7),(8), if one assumes for $E_{S}$ a value of $13 \mathrm{eV}$.

Table I summarizes the above results and Fig. 2 displays the newly found values of $C_{3}$ for the metastable atoms together with those measured earlier for groundstate atoms [3], plotted versus the static atomic polarizability.

In summary, accurate atom-surface van der Waals coefficients $C_{3}$ for the highly polarizable metastable excited $\mathrm{He}^{*}{ }^{3} \mathrm{~S}_{1}$ and $\mathrm{Ne}^{*}{ }^{3} \mathrm{P}_{2}$ atoms have been determined for the first time, using atomic diffraction off a silicon nitride transmission grating with a new theoretical approach. The experimental results are in agreement with the theory of van der Waals forces according to Lifshitz within the experimental errors. The present experimental approach can be refined to measure higher-order multipole moments of the long-range potential between the atom and the surface. In the future we plan to insert a grating into one of the beams of a Mach-Zehnder-type interferometer in order to measure atom-surface vdW potentials with interferometric precision.

We are extremely grateful to Tim Savas and Hank Smith (both MIT) for providing the $\mathrm{SiN}_{x}$ grating which has made these experiments possible. We thank L. Bruch and K. T. Tang for valuable discussions. This research has been supported in part by the Deutsche Forschungsgemeinschaft. 
[1] L. W. Bruch, M. W. Cole, and E. Zaremba, Physical Adsorption: Forces and Phenomena (Clarendon, Oxford, 1997)

[2] E. M. Lifshitz, JETP 2,1 73 (1956); I. E. Dzyaloshinskii, E. M. Lifshitz, and L. P. Pitaevskii, Adv. Phys. 10, 165 (1961)

[3] R. E. Grisenti, W. Schöllkopf, J. P. Toennies, G. C. Hegerfeldt, and T. Köhler, Phys. Rev. Lett. 83, 1755 (1999)

[4] A. Anderson, S. Haroche, E. A. Hinds, W. Jhe, and D. Meschede, Phys. Rev. A 37, 3594 (1988)

[5] W. Jhe, A. Anderson, E. A. Hinds, D. Meschede, L. Moi, and S. Haroche, Phys. Rev. Lett. 58, 666 (1987); V. Sandoghar, C. I. Sukenik, E. A. Hinds, and S. Haroche, Phys. Rev. Lett. 68, 3432 (1992).

[6] H. Failache, S. Saltiel, M. Fichet, D. Bloch, and M. Ducloy, Phys. Rev. Lett 83, 5467 (1999)

[7] M. Boustimi, B. Viaris de Lesegno, J. Baudon, J. Robert, and M. Ducloy, Phys. Rev. Lett. 86, 2766 (2001)

[8] F. Shimizu, Phys. Rev. Lett. 86, 987 (2001); F. Shimizu and J. Fujita, Phys. Rev. Lett. 88, 123201 (2002)

[9] C. S. Adams, M. Sigel, and J. Mlynek, Phys. Rep. 240, 143 (1994); O. Carnal, A. Faulstich, and J. Mlynek, Appl. Phys. B 53, 88 (1991)

[10] Y. Harada, S. Masuda, and H. Ozaki, Chem. Rev. 97, 1897 (1997); H. Hotop, Exp. Meth. Phys. Sci. 29B, 191 (1996)

[11] A. Robert, O. Sirjean, A. Browaeys, J. Poupard, S. Nowak, D. Boiron, C. I. Westbrook, and A. Aspect, Science 292, 461 (2001); F. Pereira Dos Santos, J. Léonard, Junmin Wang, C. J. Barrelet, F. Perales, E. Rasel, C. S. Unnikrishnan, M. Leduc, and C. Cohen-Tannoudji, Phys. Rev. Lett. 86, 3459 (2001)

[12] P. Engels, W. Ertmer, and K. Sengstock, Optics Commun. 204, 185 (2002)

[13] C. Fabre and S. Haroche in: Rydberg States of Atoms and Molecules, R. F. Stebbings and F. B. Dunning, eds. (Cambridge University Press, 1983).

[14] J. M. Hutson, P. W. Fowler and E. Zaremba, Surf. Sci. 175, L775 (1986)

[15] E.g., to B. Brezger, L. Hackermüller, S. Uttenthaler, J. Petschinka, M. Arndt, and A. Zeilinger, Phys. Rev. Lett. 88, 100404 (2002).

[16] R. E. Grisenti, W. Schöllkopf, J. P. Toennies, J. R. Manson, T. A. Savas, and H. I. Smith, Phys. Rev. A 61, 033608 (2000)

[17] P. Fouquet, P. K. Day, and G. Witte, Surf. Sci. 400, 140 (1998)

[18] P. E. Siska, Rev. Mod. Phys. 65, 337 (1993)

[19] C. J. Joachain, Quantum Collision Theory, 3rd Ed. (North Holland, 1983)

[20] H. Hoinkes, Rev. Mod. Phys. 52, 933 (1980)

[21] G. Vidali and M. W. Cole, Surf. Sci. 110, 10 (1981)

[22] K. T. Tang, Phys. Rev. 177, 108 (1969)

[23] Z.-C. Yan and J. F. Babb, Phys. Rev. A 58, 1247 (1998)

[24] A. Derevianko and A. Dalgarno, Phys. Rev. A 62, 062501 (2000)

[25] S. Kotochigova, E. Tiesinga, and I. Tupitsyn, Phys. Rev. A 61, 042712 (2000); M. R. Doery, E. J. D. Vredenbregt, S. S. Op de Beek, H. C. Beijerinck, and B. J. Verhaar, Phys. Rev. A 58, 3673 (1998)

[26] J. D. Jackson, Classical Electrodynamics, 3rd Edition (Wiley, New York, 1999)

[27] T. A. Savas, private communication to J. P. T.

[28] S. Zollner, E. Apen, AIP Conference Proceedings 550, D. G. Seiler et al. eds., 2001, p. 532

[29] D. M. Bishop and J. Pipin, Int. J. Quant. Chem. 47, 129 (1993); R. M. Glover and F. Weinhold, J. Chem. Phys. 66, 191 (1977) 
FIG. 1. Experimental diffraction patterns of (a) $\mathrm{He}^{*}$ at $v=2347 \mathrm{~m} / \mathrm{s}$ and (b) $\mathrm{Ne}^{*}$ at $v=873 \mathrm{~m} / \mathrm{s}$. The beam divergence of $\Delta \vartheta=0.1 \mathrm{mrad}$ and the small effective velocity spread of $\Delta v / u=3 \%$ allow ten principal maxima to be recorded. The background signal in both cases is about 10 counts/s. Solid lines have been added to guide the eye.

FIG. 2. Comparison of measured $(\times)$ and theoretical values for $C_{3}$. • : Eq. (5) with $\alpha$ from Eqs. (7) and (9); $\diamond$ : Eq. (5) with $\alpha$ exact [23]. The data points on the straight line (Hoinkes approximation [20]) are for groundstate particles [3], namely, with increasing $\alpha$ : $\mathrm{He}, \mathrm{Ne}, \mathrm{D}_{2}, \mathrm{Ar}, \mathrm{Kr}$.

TABLE I. Experimental results for $C_{3}\left(\right.$ in $\mathrm{meV} \mathrm{nm}^{3}$ ) for $\mathrm{He}^{*}$ and $\mathrm{Ne}^{*}$ compared to different theoretical expressions.

\begin{tabular}{lcl}
\hline \hline$C_{3}\left(\mathrm{He}^{*}\right)$ & $C_{3}\left(\mathrm{Ne}^{*}\right)$ & \\
\hline $4.1 \pm 1.0$ & $2.8 \pm 1.0$ & Experiment \\
$3.9 \pm 0.1$ & $3.6 \pm 0.1$ & Theory: $\alpha$ from Eqs. (8]), (6) \\
$4.0 \pm 0.1$ & - & Theory: $\alpha$ from Ref. [23] \\
\hline \hline
\end{tabular}



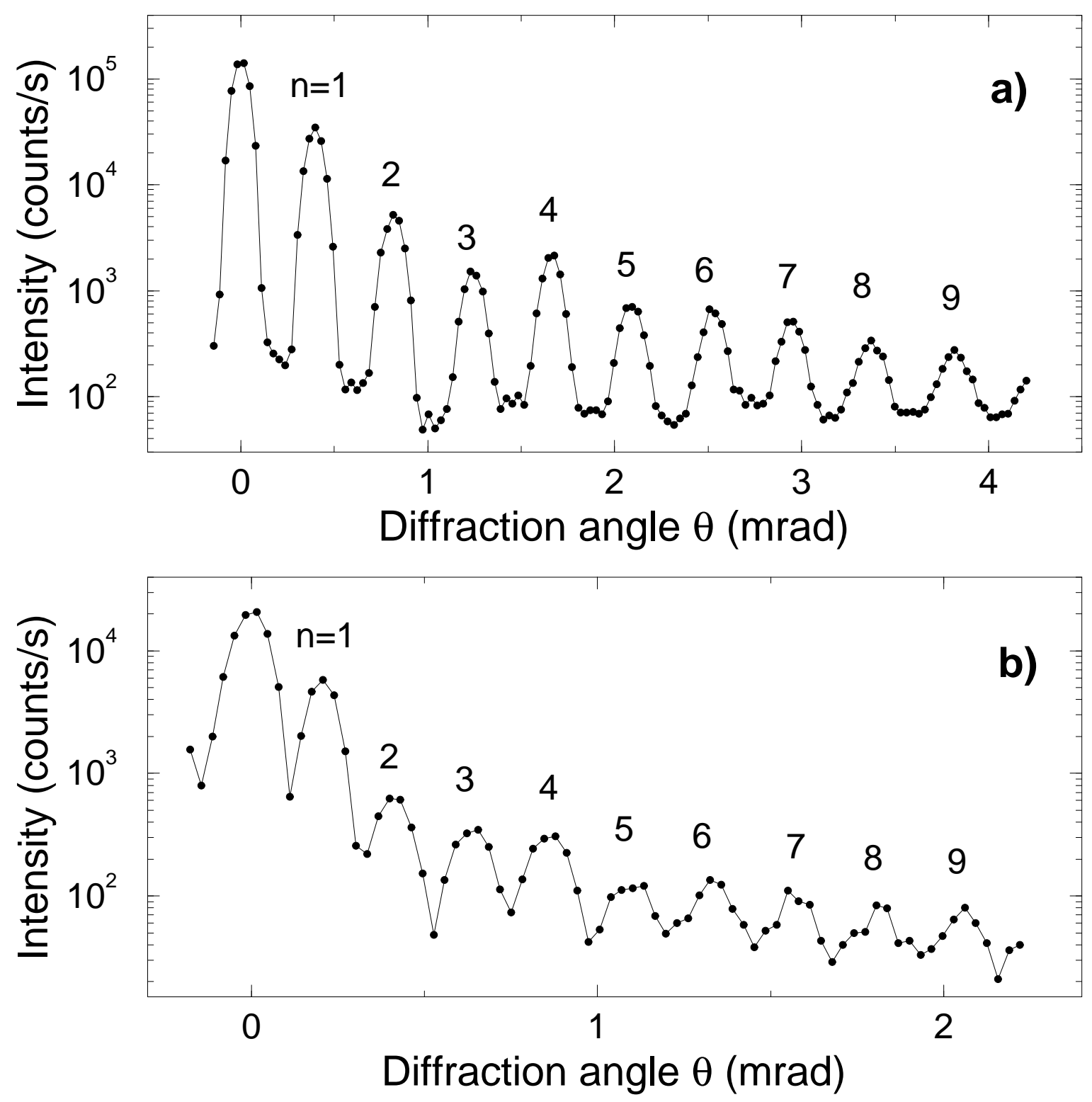


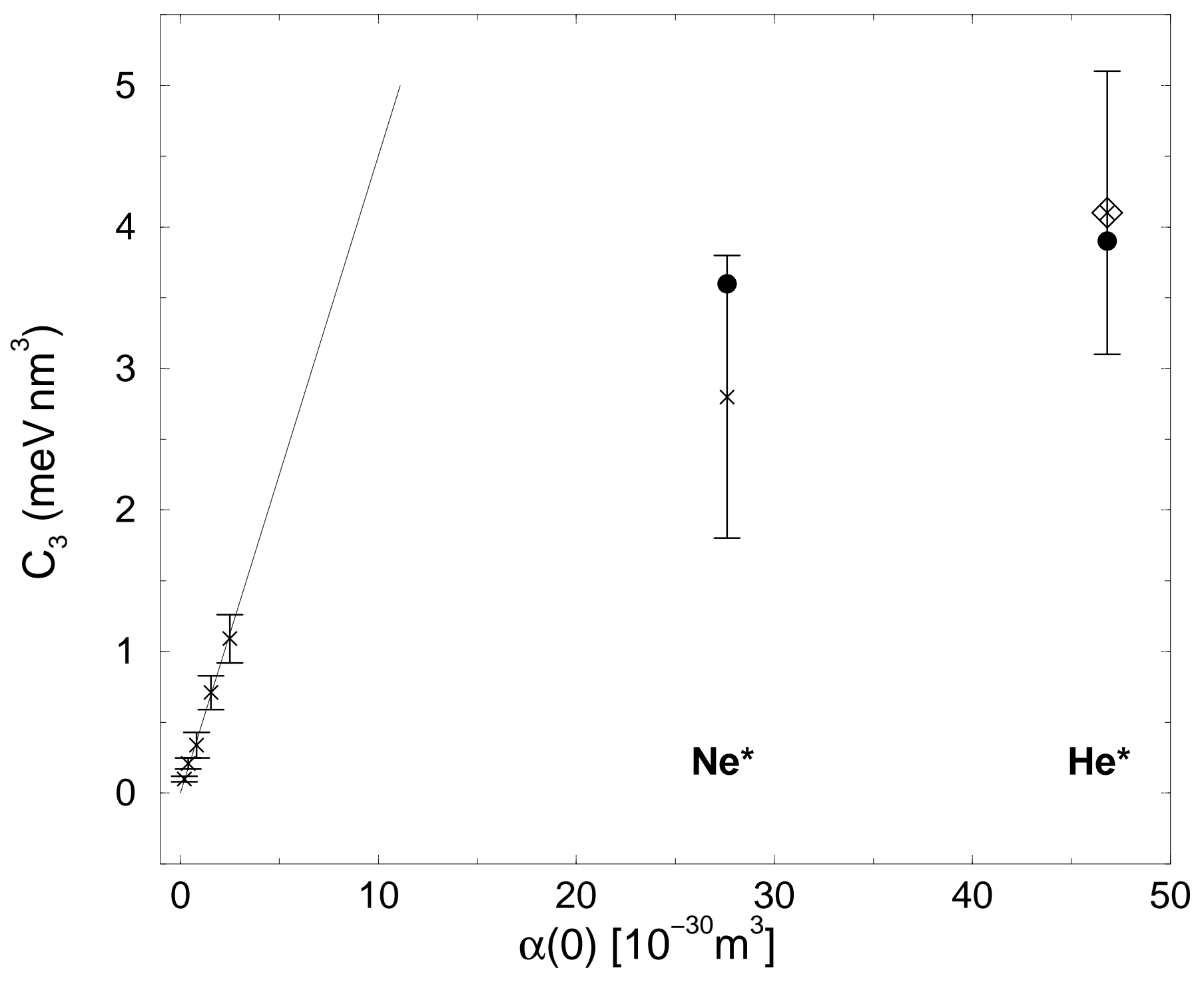

